\title{
ARTIFACTS REDUCTION IN MUTUAL INFORMATION-BASED IMAGE REGISTRATION USING PRIOR INFORMATION
}

\author{
Jundong Liu \\ School of Electrical Engineering \& Computer Science \\ Ohio University \\ Athens, $\mathrm{OH} 45701$
}

\author{
Junhong Liu \\ Nokia Inc. \\ 6000 Connection Drive \\ Irving, TX 75039
}

\begin{abstract}
Mutual information (MI) is currently the most popular match metric in handling the registration problem for multi modality images. However, interpolation artifacts impose deteriorating effects to the accuracy and robustness of MI-based methods. This paper analyzes the generation mechanism of the artifacts inherent in partial volume interpolation (PVI) and shows that the mutual information resulted from PVI is a convex function within each voxel grid. A new joint entropy estimation scheme using prior information is proposed to reduce the artifact effects and we demonstrate the improvements via experiments on misalignments between MR brain scans obtained using different image acquisition protocols.
\end{abstract}

\section{INTRODUCTION}

Image registration is one of the most widely encountered problems in a variety of fields including but not limited to medical image analysis, remote sensing, satellite imaging, optical imaging etc. Broadly speaking, image registration methods can be classified into two classes [9], namely feature-based and direct methods. Feature-based methods typically involve extracting features such as surfaces, ridges, landmark points etc., and then using a match metric to find a matching between them under a class of parameterized or more generally non-parameterized transformations. Direct methods subsume the approaches operating directly on the image grey values, without prior feature extraction.

The class of approaches based on intensity similarity measures have gained popularity in recent years. Variance of Intensity Ratio is the first and simplest statistical measure proposed by Woods et al. [11] for registering PET and MRI images. Leventon et al. [5] proposed a method based on matching the Joint Intensity Distribution of current input image with the prior joint intensity distribution obtained from training data sets.

Currently the most popular approach is based on the concept of maximizing mutual information reported in Viola and Wells et al. [10], Collignon et al. [2] and Studholme et al. [8]. For more on medical image registration algorithms, we refer the reader to the survey by Maintz et. al. [6].

Although MI methods are regarded as the best choice for the multimodal image registration problem, a number of studies have pointed out that the robustness and accuracy of MI metric is deteriorated by the interpolation artifact effects. Several methods have proposed to address this problem $[2,3,4,7]$.

This paper is a further investigation of the artifacts problem. The generation mechanism of the artifact associated with the partial volume interpolator, which is regarded as the best choice among all possible interpolators, is analyzed and correspondingly, several remedies are proposed. Experiment results using MR T1/T2 images are provided to demonstrate the improvements.

\section{MUTUAL INFORMATION METRIC AND ARTIFACT EFFECTS}

Consider two images $I_{r}(x, y)$ and $I_{f}(x, y)$. We designate $I_{r}$ as the reference image and $I_{f}$ as the floating image. Registration is to find the coordinate transformation, denoted as $T$, such that transformed floating image $I_{f}(T(x, y))$ is aligned with the reference $I_{r}(x, y)$. The alignment is usually obtained by optimizing a certain similarity metric. So normally a registration algorithm consists of three components [6]: a coordinate transform, a similarity criteria, and a numerical scheme to seek the optimum.

Mutual information is currently the most popular matching metric being used in handling the registration problem for multimodal images. The MI between two discrete random variables, $A$ and $B$, is defined as:

$$
M I(A, B)=H(A)+H(B)-H(A, B)
$$

with $H(A)$ and $H(B)$ being the entropy of $A$ and $B$, and $H(A, B)$ their joint entropy.

$$
\begin{aligned}
& H(A)=-\sum_{a} p_{A}(a) \log p_{A}(a) \\
& H(A, B)=-\sum_{a, b} p_{A B}(a, b) \log p_{A B}(a, b)
\end{aligned}
$$


where $p_{A}(a), p_{B}(b)$ and $p_{A B}(a, b)$ are the marginal probability distributions and joint probability distribution, respectively.

Given a set of samples, there are several approaches to estimate the probability functions $p_{A B}(a, b)$, most notably the histogram-based method [2] and Parzen window method [10]. In this paper, we focus on histogram-based method because it's widely used in image registration. To register the images the mutual information is to be maximized.

For digital images $I_{r}(x, y)$ and $I_{f}(x, y)$ to be aligned, interpolation is necessary to evaluate the values of $M I\left(I_{r}(x, y)\right.$, $\left.I_{f}(T(x, y))\right)$. A number of interpolators are available, including nearest neighbor (NN), linear, cubic spline, Hamanningwindowed sinc and partial volume (PV) interpolators. Among them, PV is regarded as the best choice for MI-based metrics, as pointed out by several studies $[3,7]$.

Partial volume interpolation is not an interpolation in the ordinary sense. It is a strategy being used to update the joint histogram. Instead of interpolating the new intensity value under current transformation $T$, PV directly updates the histogram of the nearest neighbor bins with the same weights used in bilinear (for 2D) or trilinear (for 3D) interpolation.

In [7], Maintz et al. qualitatively explained the reason why artifacts are generated in partial volume interpolation process and verified their arguments through several welldesigned experiments. While their work is very informative, we believe that a theoretically quantitative analysis concerning the generation mechanism of artifacts will be more instructive to guide the related research.

As interpolation affects the registration function of normalized MI and traditional MI in a similar way [7], we will construct our arguments based on traditional MI in this paper, but it should be noted that the conclusions also hold for normalized $\mathrm{MI}$.

As given above, the mutual information $M I(A, T(B))$ consists of 3 terms: $H(A), H(B)$ and $H(A, T(B)) . H(A)$ is a constant. The computation of $H(T(B))$ is also affected by the interpolation effect, but in a much smaller extent. Figure 1 shows a pair of aligned MR/CT images and the associated marginal entropies, joint entropy and MI values as functions of translations up to \pm 7 pixel distances. As evident, the variation of $\mathrm{MI}$ is dominated by the changes in $H(A, T(B)) ; H(A)$ and $H(T(B))$ are close to constants. So from now on, we will focus only on $H(A, T(B))$.

Let's First consider the situation where the reference and floating images have exactly the same pixel sizes and the motion is limited to translations only. We use the CT image in Figure 1 as the reference, while MR is the floating image. We now analyze the variation of $\mathrm{MI}$ function when the floating image moves from the alignment position to 1 pixel away along $x$ axis.

Suppose at the alignment position (translation is equal to zero), a certain histogram bin $h i s(a, b)$ has a value of
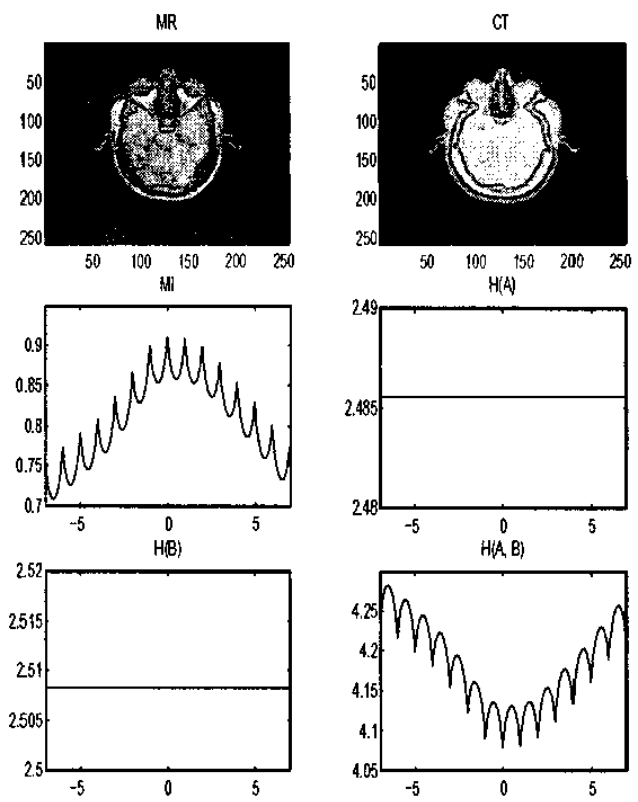

Fig. 1. The mutual information value of a pair of multimodal images. Row 1 contains a pair of MR/CT images. Row 2 and row 3 shows the mutual information(MI), marginal entropies $(H(A)$ and $H(T(B)))$ and joint entropy $((H A, T(B)))$ values as functions of translations $t$ (up to \pm 7 pixels).

$M_{1}$. The bin his $(a, b)$ records the total number of pixels in the image pair where the reference image has intensity $a$, and floating image $b$. Suppose when the translation is 1 pixel, his $(a, b)$ becomes $M_{2}$. Let's re-define the his $(a, b)$ to his $(a, b, t)$ to include the translation variable, hence his $(a, b, 0)=M_{1}$ and $h i s(a, b, 1)=M_{2}$. In between, with the translation being $t$, there are a group of intensity grids $X 1=\left\{\left(x_{1}, y_{1}\right),\left(x_{2}, y_{2}\right) \ldots\right\}$ that were originally contributing to the bin

$h i s(a, b)$, gradually wipe out their support when the floating image is moving. Let's call this group of grids as the moving-out set, and let $A_{1}$ be the total number of these grids. Because the motion here is limited to translation only, all the grids in the moving-out set are withdrawing their contributions to the bin $h i s(a, b)$ at the same rate, as the translation increases from 0 to 1 . When the translation is 0 , each of them contribute an ' 1 ' to his $(a, b)$; when the offset is 1 , they do not have contribution any more. In between, the contribution of the each moving-out grid is $1-t$.

Similarly, there might be another group of grids $X_{2}$ (let $A_{2}$ be the total number) that were not originally contributing to his $(a, b)$, start moving in to contribute to his $(a, b)$ as the translation increases from 0 to 1 . Their individual 
contribution to his $(a, b)$ is ' $t^{\prime}$ at translation $t$.

Overall, the combined effects of the moving-in and movingout sets lead to the change of his $(a, b)$. So we have: $A_{2}-$ $A_{1}=M_{2}-M 1$. At translation t:

$$
\begin{aligned}
\operatorname{his}(a, b, t)=M_{1}+A_{2} t-A_{1} t & =M_{1}+(M 2-M 1) t \\
& =t M_{2}+(1-t) M_{1}
\end{aligned}
$$

So basically within interval $[0,1]$, the bin value $h i s(a, b, t)$ is a linear function of the offset variable $t$, denoted here by $f(t): f(0)=M 1, \quad f(1)=M 2$,

$$
f(t)=t f(1)+(1-t) f(0) .
$$

Since we use histogram to approximate distribution, the joint entropy of two images can be rewritten as $H(A, T(B))=$ $-\sum_{a, b} h i s(a, b, t) \log h i s(a, b, t)$. As we know, the function $x \log x$, denoted by $g(x)$ here, is a convex function within the interval $(0,1]$ (note its second derivative is positive), i.e.:

$$
g\left(t x_{1}+(1-t) x_{2}\right) \leq t g\left(x_{1}\right)+(1-t) g\left(x_{2}\right)
$$

Therefore, the individual contribution of the bin his $(a, b, t)$ to the joint entropy $H(A, T(B))$ follows:

$$
\begin{aligned}
g(f(t)) & =g((1-t) f(0)+t f(1)) \\
& \leq(1-t) g(f(0))+t g(f(1))
\end{aligned}
$$

The inequality above indicates that each component of $H(A, T(B))$ is a convex function within $[0,1]$. Since the summation of convex functions is still a convex function, $\mathbf{H}(\mathbf{A}, \mathbf{T}(\mathbf{B}))=-\sum \sum \mathbf{g}(\mathbf{h i s}(\mathbf{a}, \mathbf{b}, \mathbf{t}))$, as a negative combination of certain number of convex functions, is a concave function in $[0,1]$. Correspondingly, the $\mathrm{MI}$ responses is a convex function in the same interval. This property can be easily extended the any intervals $[n, n+1]$ where $n$ is an integer. That's the reason the responses of $H(A, T(B))$ as a function of translation $t$ (Figure 1 ) bears a concaveshaped artifact within each integer interval.

The above analysis indicates that the heart of the artifacts generation mechanism lies in the following fact: all the moving-in and moving-out grids contribute to the change of the bin value at a synchronized pace. As a consequence, a general guideline to reduce the artifact effects can be "to break the synchronization".

In addition, the following prediction can be made based on the above analysis:

- The artifact effect for pure rotations would be less severe than that of pure translations. This is because the moving-in and moving-out grids, under the pure rotation motion scenario, do not contribute to the change of histogram in a uniformly rate. Figure 2.a. shows the $H(A, T(B))$ values as a function for rotations (up to $\left.\pm 15^{\circ}\right)$. As evident, the responses for rotations are much smoother than the translation counterpart.
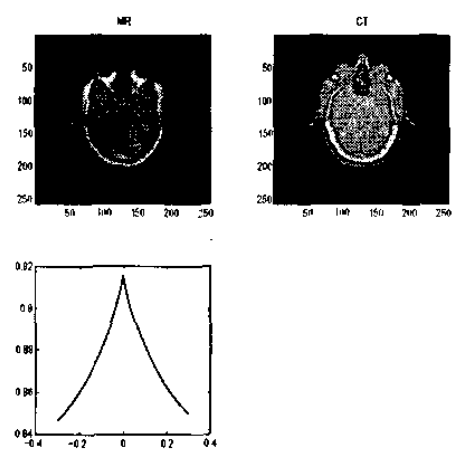

Fig. 2. Row 1 contains a pair of MR/CT images. Row 2 shows the mutual information (MI) value as a function to rotaions $\left( \pm 20^{\circ}\right)$.

\subsection{Artifacts Reduction}

Based on the abolve analysis, we propose the an artifact effects reduction scheme based on integrating prior information. The idea is to wipe out the concave function portion of the joint entropy, with the linear function part kept. This task can be mostly done by combining the joint entropy $H(A, T(B))$ with a prior joint entropy $H^{*}(A, T(B))$. The justification is based on the assumption that the training data would provide a prior joint probability that is similar to the probability of the test data, therefore the artifact part can be effectively removed by subtracting the concave function part of the prior joint entropy. To achieve this end, we replace the MI metric with a modified version: $\bar{M} I(A, T(B))=H(A)+H(T(B))-(\bar{H}(A, T(B))$

Suppose $T$ is a $2 \mathrm{D}$ rigid transform vector $\{\alpha, d x, d y\}$, let

$$
\begin{aligned}
& T_{00}=\{\alpha, \text { floor }(d x), \text { floor }(d y)\} \\
& T_{01}=\{\alpha, \text { floor }(d x), \text { ceil }(d y)\} \\
& T_{10}=\{\alpha, \text { ceil }(d x), \text { floor }(d y)\} \\
& T_{11}=\{\alpha, \text { ceil }(d x), \text { ceil }(d y)\}
\end{aligned}
$$

where floor and ceil stand for floor and ceiling operations respectively. The modified joint entropy $\bar{H}(A, T(B))$ is defined as follows:

$$
\begin{array}{r}
\bar{H}(A, T(B))=H(A, T(B))-H^{*}(A, T(B)) \\
+\quad \text { BiLinear }\left(H^{*}\left(A, T_{00}(B)\right), H^{*}\left(A, T_{01}(B)\right),\right. \\
\left.H^{*}\left(A, T_{10}(B)\right), H^{*}\left(A, T_{11}(B)\right)\right),
\end{array}
$$

where BiLinear is the bilinear interpolation operation.

Figure 3 depicts the registration for the new MI function. The top left subfigure of Figure 3 shows the MI artifact patterns for the CT/MR test image. The top right shows the concave function that is obtained from a pair of registered training images. As evident in the bottom subfigure, a significant reduction of interpolation artifacts was achieved after subtracting the concave function. 

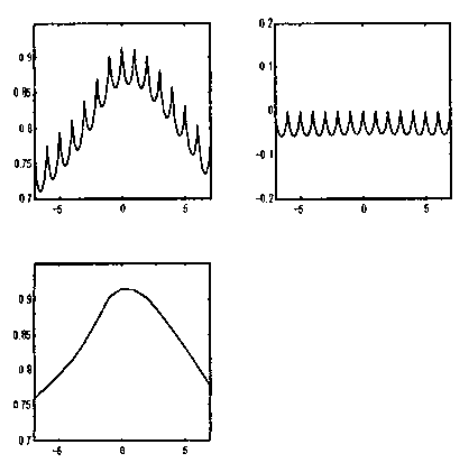

Fig. 3. Top left: The MI artifact patterns for the CT/MR image pair. Top right: The prior concave function to be subtracted. Bottom left: $\bar{M} I$ responses after substracting the prior concave function.

\section{EXPERIMENT RESULTS}

In this section, we demonstrate the robustness property of the new MI computation method proposed in the previous section. All the examples contain synthesized miss-alignments applied to MR data sets from the brainweb site at the Montreal Neurological Institute [1].

The experiments are designed as follows: with a 2D MR T1 slice as the reference image, the floating image is obtained by applying a rigid transformation to a previously aligned 2D MR T2 image.

With 15 randomly generated rigid transformations, we applied our Integrating Prior Joint Entropy algorithms together with the traditional MI method to estimate motion parameters. These transformations are normally distributed around the values of $\left(0^{\circ}, 10\right.$ pixel, 10pixel $)$, with standard deviations of $\left(5^{\circ}, 3\right.$ pixel 3 pixel $)$ for rotation and translation in $x$ and $y$ respectively.

Table 1 depicts the mean and standard deviation of the estimation errors obtained from the 2 methods. In each cell, the leftmost value is the rotation angle (in degrees), while the right two values show the translations in $\mathrm{x}$ and $y$ directions respectively. Out of the 15 trials, the traditional MI failed 5 times while the Integrating Prior Joint Entropy never failed ("failed" here means that the results had unacceptably large errors). If we only count the cases which gave reasonable results, as shown in the first (for Integrating Prior Joint Entropy) and third (for traditional MI) rows, our approach and the traditional MI have comparable performances, all being very accurate. Note that Powells method was used as the optimization scheme in these experiments.

\begin{tabular}{|l|lll|lll|}
\hline & \multicolumn{3}{|c|}{ mean } & \multicolumn{3}{c|}{ standard deviation } \\
\hline 1 & $0.132^{\circ}$ & 0.293 & 0.372 & $0.012^{\circ}$ & 0.112 & 0.230 \\
\hline 2 & $0.087^{\circ}$ & 0.293 & 0.383 & $0.031^{\circ}$ & 0.121 & 0.129 \\
\hline
\end{tabular}

Table 1. Comparison of estimation errors for rigid motion between Integrating Prior Joint Entropy and traditional MI.

\section{CONCLUSIONS}

In this paper, we quantitatively analyzed the generation mechanism of the interpolation artifacts. Two remedies: Slightly Rescaling and Including Prior Joint Entropy were proposed to reduce the artifact effects. Comparisons were made between the traditional MI and the two modified MI implementations. Experimental results depicted better performance of using the modified method over the traditional MI.

\section{Acknowledgments}

We thank the brainweb data source creators at the Montreal Neurological Institute for the data.

\section{REFERENCES}

[1] Simulated brain database [Online]. Available : http://www.bic.mni.mcgill.ca/brainweb/

[2] A. Collignon, F. Maes, D. Delaere, D. Vandermeulen, and P. S. ang G. Marchal, "Automated multimodality image registration using information theory," Proc. IPMI,Y.J.C.Bizais, Ed., pp. 263-274,1995.

[3] X. Ji, H. Pan and Z.P. Liang, "A region-based mutual information method for image registration", Proc. 7th Intl. Soc. Magn. Reson. Med., vol 3, pp 2193, May, 1999.

[4] B. Likar and F. Pernus, "A hierachical approach to elastic registration based on mutual information", Image and Vision Computing, 19, 2001, pp. 33-44.

[5] M. Leventon and W. E. L. Grimson, "Multi-modal volume registration using joint intensity distributions," in MICCAI 1999.

[6] J.B. Maintz and M. A. Viergever,"A Survey of Medical Image Registration," MedIA Vol. 2, pp. 1-36,1998.

[7] J. Pluim, J. Maintz and M. Viergever, "Interpolatioin artefacts in mutual information-based image registration," Computer Vis Image Underst, vol. 77, pp 211-232, 2000.

[8] C. Studholme, D. Hill and D. Hawkes, "An overlap invariant entropy measure of 3D medical image alignment," Pattern Recognition, Vol. 32, pp. 71-86,1999

[9] B. C. Vemuri, S. Huang, et.a.l., "An efficient motion estimator with application to medical imaging," Med. Image Anal., 2(1), pp. 79-98, 1998.

[10] W. M. Wells III, P. Viola, and H. Atsumi, "Multi-modal Volume Registration by Maximization of Mutual Information," Medical Image Analysis, 1(1), pp. 35-51, 1997.

[11] R. Woods, J. Maziotta and S. Cherry "MRI-PET registration with automated algorithm," J. Comput. Assis. Tomogr, 17, pp. 536-546, 1993. 